\title{
Photoreceptors and Control of Horticultural Plant Traits
}

\author{
Kevin M. Folta ${ }^{1}$ and Sofia D. Carvalho \\ Horticultural Sciences Department, University of Florida, Gainesville, FL; and Plant Molecular and Cellular \\ Biology Program, University of Florida, Gainesville, FL
}

\author{
Additional index words. LED light, photoreceptors, controlled environment, artificial light, photomorphogenesis
}

\begin{abstract}
Plant productivity and product quality ultimately are dependent on an interaction between genetics and environment, and one of the most important environmental cues is light. Light quantity, quality, and duration provide critical information to plants that mediate growth and development. Light signal transduction is dependent on a series of photoreceptors and their associated signaling pathways that direct intracellular processes that lead to changes in gene expression that ultimately affect plant form, function, and content. For the last several decades, scientists have dissected these signaling pathways and understand how they connect the environment to a response. The advent of narrowbandwidth illumination in commercial lighting invites the opportunity to manipulate plant behavior and productivity through precise alteration of the ambient spectrum. This review describes the biochemical links that convert incident light into predictable changes in plant growth and development. These sensors and pathways serve as biochemical switches that can be selectively toggled to control plant growth, development, physiology, or metabolite accumulation.
\end{abstract}

Over the last decade there has been significant effort in improving narrow-bandwidth, solid-state lighting systems for plant growth in controlled environments. Development of the electronics and light sources has followed the convention that plants can survive well under lights designed for the human eye, with only minor consideration of empirically defined plant needs. In addition, the emphasis on simply growing plants has ignored the real opportunity to control the way plants grow, and neglected the fact that light can be a means to affect expression and variation of high-value plant traits.

A plant phenotype is an output shaped by genetics and environment. Connection between environment and genetics begins with activation of several sensitive photopigments that constantly scan the plant's ambient environment. One of the most important and influential environmental signals is light. Changes in light conditions lead to alterations in morphology, gene expression, physiology, and metabolism. In photosynthetic organisms light is the fundamental driver of autotrophy. But light is much more than the energy of metabolism - it is a packet of precise information that describes the immediate environment with respect to time and space. Over the past few decades it has been demonstrated that discrete sections of the electromagnetic spectrum tickle specific photoreceptors that then ignite plant sensory networks, ultimately resulting in a change in content, stature, or behavior.

These concepts underlie the exciting opportunity of "Plant Whispering," commanding a plant what to do and when to do it, based on a vocabulary of signals the grower can control. The plant photosensor collection is listening for light cues. Photons from various portions of the spectrum excite different receptors, each controlling specific facets of plant biology. Manipulating the information within an ambient spectrum may be seen as

This work was performed with funding from the National Science Foundation Grant IOS-0746756. 1'Corresponding author. E-mail: kfolta@ufl.edu. an opportunity to shape plant quality and productivity with guiding instructions. We are reminded that plants are environmentally modified organisms, and environmental control has the potential to predictably adjust plant traits within a plant's genetic potential, and can produce desirable changes in a much shorter time than breeding or other genetic modification.

This concept is not new. Researchers have long known that manipulation of the light spectrum can be used to control specific aspects of plant growth. Previous work has successfully used different light sources with skewed spectra relative to solar radiation (Islam et al., 2012), colored shade cloths to change plant attributes (Stamps, 2009; Takeda et al., 2010), colored mulches that alter reflected sunlight (Loughrin and Kasperbauer, 2003), or even Christmas tree lights draped in the plant canopy (Takeda et al., 2010). Only now is it possible to realistically devise a custom plant spectrum from an electronic canopy that actively changes to satisfy grower demands rather than simply facilitating baseline plant needs.

The central theme of this review is to outline how plants sense light, and how those signals translate into predictable and controllable outputs in plant biology. We recently reviewed the scholarly literature on narrowbandwidth light effects on plant growth and development (Carvalho and Folta, 2014a). The work described the effects of growing a range of crop plants under narrowbandwidth radiation, such as that provided by LED light. To complement that work, this review addresses the range of sensors that funnel photons of discrete energy into specific transduction cascades, resulting in changes in physiology and gene expression, building on the concept that light can be used as a growth regulator (Folta and Childers, 2008). We can approach the compelling hypothesis that LED-based light sources present an opportunity to actively direct plant growth, development, and metabolite accumulation. We are whispering to plants with the language of light, and LED lights allow us to control that vocabulary.

\section{PLANT PHOTOSENSORS}

Responses to light begin with activation of photoreceptors that are sensitive to specific wavelengths of light. Think of the plant light sensors as a series of switches that may be toggled with different light wavelengths. More precisely, they are a series of switches and dimmers, each modulating the activity of a network of circuits that control specific processes that affect plant output. These circuits are sensitive to not only the wavelength, but also the fluence rate ( intensity) and photoperiod. Together they interact with each other, and their combined interaction influences the final plant product quality, whether it is the color of lettuce leaves or phytonutrients in sprouts.

The plant photoreceptors are proteins connected to a light-sensing chromophore, a molecular antenna that absorbs a photon of specific wavelength, jockeys around some electrons, and then induces conformational changes in the receptor protein that complete the conversion of electromagnetic energy into biochemical potential. The chromophore and protein together have specific lightabsorption properties, and this is the basis behind fine control of plant physiology, development, or metabolite accumulation.

The plant light sensors and a depiction of their approximate sensitivities to electromagnetic energy are shown in Fig. 1. From left to right, arranged from short wavelengths to long, there are sensors that react to ultraviolet light all the way through the visible spectrum, and ending with sensitivities in the far red portion of the spectrum. The range of the plant light spectrum well eclipses the rainbow that defines the limit of human vision. Specific plant responses have been attributed to ultraviolet-B, blue, green, red, and far red wavelengths. Responses have been noted as far out as ultraviolet-C (260 nm; Hamner et al., 2009) and even into the near infra-red (Johnson et al., 1996).

The wide span of spectral sensitivity is consistent with what we know about plants and their evolution. As organisms anchored to the earth, survival is aided by mechanisms 


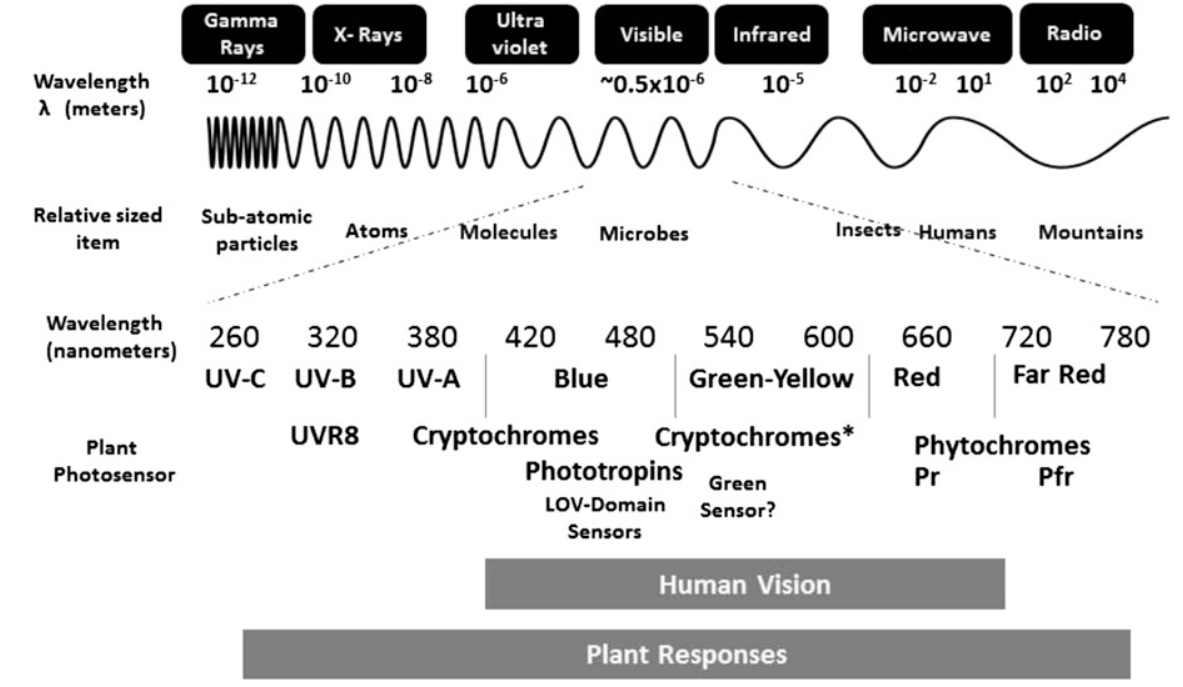

Fig. 1. The relative distribution of electromagnetic energies and the wavelengths that discretely interact with plant photosensors.

to sense and adapt to environmental change. Sunlight is composed of a wide set of wavebands, some that reach the earth's surface. These energies vary in intensity and relative ratio based on time of day, latitude, and season. The various wavebands are differentially transmitted or absorbed by plant materials. Therefore it only stands to reason that plants would evolve mechanisms to capture and interpret these signals. The range is limited on the short wavelength side by a paucity of short-wavelength energies that can penetrate the earth's atmosphere. On the long-wavelength side quantum energies are insufficient to excite photochemical responses, or are too long to stimulate molecular chromophores. Organisms sensing energies into this range are pit vipers (Gracheva et al., 2010) and vampire bats (Gracheva et al., 2011) that rely on specialized physical structures with no known analogs in plants.

The individual sensors were predicted from years of careful observation of plant physiology and developmental behaviors, and extensive reviews on the subject have been published (Briggs and Christie, 2002; Chen et al., 2004; Lin, 2000). Over the last decade the effect of light on horticultural traits and the increased availability of narrow-bandwidth light sources present an opportunity to exploit our knowledge of light-sensory circuitry to custom blend light regimens that best drive plant responses to match grower needs.

\section{PHYTOCHROMES}

Many processes, from control of plant stature all the way to fundamentals of gene expression, are under the influence of phytochrome control. These pigments are sensitive to red and far red light, but also have absorption in the blue/near-ultraviolet. Phytochromes accumulate in the cytosol in darkness. Stimulation of phytochrome pigments by light initiates a series of events both in the cytoplasm and in the nucleus. The activated photosensors direct cytosolic changes in prothe nucleus where they alter gene expression events. Many of these are related to alterations in hormone levels, and effects on gibberellins (Feng et al., 2008), auxins (Tao et al., 2008), ethylene (Khanna et al., 2007), jasmonates (Moreno et al., 2009), and ABA (Piskurewicz et al., 2009) have been well described for decades (Mohr, 1962; Pjon and Furuya, 1967; Vince, 1964). These changes in growth regulators then mediate alterations in plant morphology, physiology, development, and metabolism.

Phytochromes offer a unique opportunity to control processes related to plant productivity, mostly because the connections between specific phytochromes and downstream responses have been well characterized for at least a subset of phytochrome isoforms. Each plant genome contains a number of different phytochromes, each expressed in different patterns and possessing varying ability to interact with each other (Sharrock and Clack, 2004). Specific members of the phytochrome family are more stable and active under far red conditions. These far red-active phytochromes (belonging to the Type I or "phyA" type) have specific downstream molecular targets and control discrete processes after illumination with far red light $(\approx 730 \mathrm{~nm})$. They are typically extremely sensitive, react to even low-fluence-rate visible light within minutes, and are abundant in dark-grown tissue. They are most aptly characterized in their responses during de-etiolation, yet they play central roles in later plant life, such as in the control of flowering (Chen et al., 2010; Valverde et al., 2004).

Other phytochromes, the Type II phytochromes, show a characteristic switching The red-absorbing form is activated by photons of red light $(\approx 660 \mathrm{~nm})$ leading to various downstream changes in gene expression and tein phosphorylation, as well as localize to ability between active and inactive forms. cellular responses. The most important characteristic in these responses from a plantcontrol standpoint is the ability to attenuate a red light response using a far red light treatment (Furuya and Torrey, 1964; Kaufman et al., 1985). These red-activated phytochromes are also found in the cytosol and move the nucleus slowly, in hours rather than minutes (compared with phyA). They are important for sustained responses to light and play roles from shade avoidance responses to participation in flowering control.

The central mechanism of phytochrome function is in control of nuclear gene expression. Phytochromes control gene expression, at least in part, by negatively regulating the repression imparted by the COP/DET/FUS proteins, leading to accumulation of transcriptional activators such as HY5, HYH, LAF1, and HFR1 (Duek et al., 2004; Osterlund et al., 2000; Zhu et al., 2008). In darkness, these positive-acting transcriptional regulators are degraded via ubiquitination and the $26 \mathrm{~S}$ proteasome. Activation of phytochrome (and other light sensors) changes the rate of degradation, allowing transcriptional activators to accumulate.

Phytochromes do not only inactivate repressors, there is a substantial body of evidence that indicates a role as positive regulators in conjunction with phytochrome-interacting factors, or PIFs. PIF interaction with phytochromes is light-dependent and reversible (Leivar et al., 2008). It has also been shown that PIF3 interaction with phytochrome $\mathrm{B}$ leads to photoreversible interaction with promoters containing specific DNA binding elements (Martinez-Garcia et al., 2000). The phytochrome A receptor also has been shown to interact with specific DNA binding elements in chromatin immunoprecipitation assays (Chen et al., 2014), and is known to directly participate in chalcone synthase expression by interacting with PIF3 at the promoter (Chen et al., 2012). Overall, it appears that phytochromes exert their main influence via control of transcription networks, and do so depending on the relative ratio of red and far red illumination.

However, when we consider application, many commercial horticultural lighting fixtures, especially the new LED-based fixtures, do not feature specific far red light emitters. Certainly incandescent and other broadspectrum sources can include these wavebands. This absence in narrow-bandwidth source leaves a clear opportunity to control plant behaviors or product qualities essentially unexploited. An extensive body of basic research shows that phytochromes are central in controlling plant stature, pigmentation, and developmental transitions like flowering. These are all important attributes of commercial plants and may be manipulated through activation and inactivation of specific phytochromes using red and far red light.

One example is the use in potentially promoting elongation growth using endof-day (EOD) far red treatments. It has been demonstrated that after darkness, even a brief pulse of far red light can evoke profound 
effects on plant stature. It was shown that a 5-min pulse of far red light can alter elongation and chlorophyll content in tobacco leaves (Kasperbauer and Peaslee, 1973), and that the effect is transduced through phyB (Reed et al., 1993). Previous work on controlling plant behaviors with skewed spectrum has typically relied on the use of specific films to alter the spectrum for EOD treatments (Ilias and Rajapakse, 2005).

The use of EOD far red from LED supplementation has been implemented to induce elongation of cucurbit (Yang et al., 2012) and tomato (Chia and Kubota, 2010) plants before grafting. Far red EOD treatments were also examined in controlling elongation growth of chrysanthemum (Lund et al., 2007). Elongation growth in stems and leaves may be desirable in specific crops, and a simple EOD far red treatment, transduced through the phytochrome system, may be a simple means to control plant product attributes.

Further exciting approaches using phytochromes to control plant growth habits can make use of simultaneous irradiation of red and far red light. Different combinations of the wavelengths give the opportunity to generate ranges of equilibrium that can promote/repress traits of interest to specific extents. While most current narrow-bandwidth sources do not include far red emitters, it is almost certain that attention to the potential manipulation of phytochrome responses by far red light will warrant addition of these electronics to lighting designs.

\section{CRYPTOCHROMES}

The blue-light sensing cryptochrome receptors (cry1 and cry2) were identified because they had profound effects on seedling development (Ahmad and Cashmore, 1993) and flowering (Guo et al., 1998). The proteins bear greatest similarity with photolyases, light-activated enzymes that reverse DNA damage using light as an energy source, yet the cryptochromes have no DNA-repair activity (Sancar, 2003). Cryptochromes use two chromophores, a folate and flavin adenine dinucleotide (FAD). These receptors have many roles in plant growth and development, mostly mediating responses to higher-fluencerate $\left(>50 \mu \mathrm{mol} \cdot \mathrm{m}^{-2} \cdot \mathrm{s}^{-1}\right)$ blue and ultraviolet-A light (Lin, 2002).

Cryptochromes mediate a variety of plant growth responses. While most well known for their roles in deetiolation and photoperiodic flowering control, the cryptochromes have been shown to entrain the circadian clock (Devlin and Kay, 2000; Somers et al., 1998), control root growth (Canamero et al., 2006), plant height (Giliberto et al., 2005; Platten et al., 2005), and fruit size (El-Assal et al., 2004). They also have been shown to participate in guard cell opening (Mao et al., 2005), and stem elongation (Chatterjee et al., 2006; Folta and Spalding, 2001). They also are important in controlling pigmentation through the accumulation of anthocyanins (Chatterjee et al., 2006; Fox et al., 2012) as well as a role in regulating transcriptomic responses to high light stress (Kleine et al., 2007).

Cryptochromes also are central to the regulation of the flowering transition. The blue-light contribution through cry2 was demonstrated specifically in Arabidopsis where cry 2 activation induces accumulation of proteins that stimulate the floral transition (Valverde et al., 2004). In tomato, overexpression of cry 2 does the opposite and delays flowering, yet induces emergence of axillary branches (Giliberto et al., 2005). In other crops the cry receptors promote flowering, such as in rice (Hirose et al., 2006). Blue light, most likely acting through cryptochromes, induces flowering in important flowering species like chrysanthemum (Khattak and Pearson, 2006) or Morning Glory (Yamazaki et al., 2007).

The flavin chromophore in cryptochromes assumes transient redox states that affect its absorption properties. The semireduced flavosemiquinone is a green absorbing form that has been shown to impart biological outcomes in Phycomyces (Galland and Tolle, 2003) and Arabidopsis (Bouly et al., 2007). The change in redox state is wavelength dependent, meaning that a blue-light treatment will induce accumulation of the semireduced or fully reduced chromophore. These changes may be reverted using green light, reversing the blue-light response, much as phytochrome responses may be modulated with red and far red light treatments.

This cryptochrome-mediated, blue-green reversibility was demonstrated in Arabidopsis. The action spectrum for cry1 responses shows a maximum effect at $450 \mathrm{~nm}$ (Ahmad et al., 2002). The addition of green light at $563 \mathrm{~nm}$ can inhibit the blue-light response, as well as the blue-light mediated destabilization of the cry 2 protein (Bouly et al., 2007). Green light has been shown to repress flowering (Klein et al., 1965; Vince et al., 1964). Banerjee et al. (2007) show that green light can inhibit flowering in Arabidopsis, a process known to be strongly influenced by blue light acting through cry2 (Valverde et al., 2004).

The cryptochromes physically and functionally interact with phytochromes in regulating many of these roles, mechanistically defining the phenomenon of "coaction" (discussed below). In the design of future narrow-bandwidth lighting systems, there is substantial opportunity in strategic mixes of red, blue, and far red light to tune signal flux through phytochromes and cryptochromes. It should be noted that cryptochrome responses tend to be high-fluence-rate responses, so they may be coarsely separated from the effects of other blue-light sensors by controlling light intensity.

\section{PHOTOTROPINS}

The phototropins are blue-light photosensors that represent another route that these signals may contribute to shaping plant form and function. Blue-light signals result in a variety of changes from the whole plant to the accumulation of specific transcripts. Unlike the general effects of cryptochromes, the phototropins guide a wide variety of plant responses that tend to share a common themethey all are directly or indirectly related to optimizing photosynthesis.

It has long been observed that plants bend toward light, and these movements are stimulated well by blue light. Throughout the 1980s and 1990s a series of biochemical and genetic analyses began narrowing down the search for the blue-light sensors that coordinated phototropic growth. Analysis of phototropic mutants led to the cloning of a gene encoding an autophosphorylating serine/threonine kinase that used flavin mononucleotide as a cofactor (Christie et al., 1998). This molecule (phot1) was shown to initiate the response to blue light, even dim pulses, which would lead to changes in plant growth and development. Later, a paralogous gene with discrete functions was cloned and characterized as phot2.

Genetic analyses have defined roles for phototropins in a wide series of plant responses. Aside from their namesake phototropic behaviors, phototropins play roles in controlling leaf expansion in lowlight (Takemiya et al., 2005) and in leaf position (Inoue et al., 2008). Whereas red light and phytochrome B promote leaf curling, phots repress that response, leading to flatter leaves and a more efficient surface for light absorption (Kozuka et al., 2013). The role of blue light and phototropin influence offer an opportunity to translate the findings from Arabidopsis to controlled environment crop plants. It may be possible to use low-fluence blue light to encourage rapid expansion of leaf materials early in development (Takemiya et al., 2005), to present larger surfaces for photosynthetic source tissue later, or even larger leaves for use as food. Phototropin1 also has a role in suppressing lateral root growth (Moni et al., 2014), an important trait for many food crops being grown in artificial environments. Phototropins also have been shown to affect the accumulation of anthocyanins in strawberry (Kadomura-Ishikawa et al., 2013), a response that is typically controlled by cryptochromes in vegetative tissues.

Aside from these conspicuous effects on leaves and roots, phototropins are central in nuanced cellular responses to the light environment, and as mentioned previously, these seem to center around optimizing photosynthesis (Spalding and Folta, 2005). These include control of chloroplast accumulation and avoidance responses, where the organelles align perpendicular to the plane of incident light under lowlight conditions, yet move to parallel positions in a highlight environment (Kagawa, 2003). Phototropins also regulate the stomatal opening (Boccalandro et al., 2012) and accumulation of specific mRNAs associated with photosynthesis (Folta and Kaufman, 2003). They also have influence on how blue-light signals are integrated in the shade environment (Keuskamp et al., 2011). 
With regard to design of artificial lighting environments, flux through phototropins is critical to consider. A failure to provide sufficient blue-light activation of phototropins results in the aforementioned shade avoidance responses, which may, or may not be desirable in manipulating qualities of a given fruit or vegetable crop product. The main consideration for phots will be in the optimization of plant growth and development to accommodate photosynthesis, as phot receptors seem to be central in tailoring the form and function for this process.

\section{OTHER LOV-DOMAIN RECEPTORS}

The LOV domain (for "Light, Oxygen, Voltage," named for its roles in prokaryotes) is the functional activation sequence of the PHOT protein. Shortly after roles for the PHOTs were elucidated and mechanism of action defined, other blue-light sensors were identified, also containing this activation motif. Three non-PHOT, LOV domain proteins were identified in Arabidopsis, FKF, LKP2, and ZEITLUPE (ZTL). They have roles in conditioning the circadian oscillator (Nelson et al., 2000; Schultz et al., 2001; Somers et al., 2000), and affect developmental regulators, such as those that mediate the transition to flowering.

FKF1 was isolated from a pool of lateflowering mutants, rescued by vernalization or gibberellin treatment (Nelson et al., 2000). Together with ZTL it stabilizes CONSTANS for proper control of flowering, through a mechanism enhanced by blue light (Song et al., 2012, 2014). ZTL also acts with LKP2 in the presence of blue light to suppress degradation of the floral initiator cryptochrome-interacting basic helix-loop-helix 1 (CIB1), by a process independent of cry2 activity (Liu et al., 2013).

Because these chromoproteins have such specific effectors, they may be prime targets for control of discrete processes, like flowering. However, their roles are typically dependent on the upstream activities of other photosensors with overlapping light sensitivities. Therefore, the LOV domain receptors may be better genetic targets for mutation or editing rather than for environmental manipulation.

\section{UVR8}

Ultraviolet light is efficiently screened from sunlight as it passes through the earth's atmosphere. However, even the reduced quanta of short-wave energies that pass through have the potential to cause harmful effects on terrestrial organisms through damage of proteins or DNA, peroxidation of lipids, or through other effects of reactive oxygen species. Plants, evolving under a constant bombardment of these threats without the ability to move away, have evolved molecular and physiological mechanisms to adjust to or repair damage from ultraviolet light. Ultraviolet light can be categorized as ultraviolet-A $(315-400 \mathrm{~nm})$, ultraviolet-B (280-315 nm), and ultravioletC (230-280 nm). Effects of ultraviolet-B radiation have been shown to cause discrete photomorphogenic effects on plants, and it has long been posited that an ultraviolet-B sensory system is in place transducing those effects.

Ultraviolet-B treatment has conspicuous consequences in terms of plant architecture and pigmentation. These wavelengths primarily limit extension growth, leading to plants that are more compact in stature. Leaf expansion is limited, and there is a tendency to favor branching over stem elongation (Frohnmeyer and Staiger, 2003). These traits are distinct from those induced by visible wavebands, all have potential to add value in certain horticultural crops, and illustrate the potential for ultraviolet-B application to be a valuable component in controlled environments.

At lower fluence rates, ultraviolet-B generates changes in gene expression that induce production of photoprotective pigments (such as the anthocyanins) and damagerepair mechanisms. For instance, irradiation with UVB increases transcript levels of CHALCONE SYNTHASE and the ELONGATED HYPOCOTYL 5 transcription factor, the former central to the phenylpropanoid pathway and the latter a potent agent of photomorphogenic behaviors. Ultraviolet-B irradiation also induces networks associated with stress, wounding, and defense (Brosche and Strid, 2003; Kilian et al., 2007). Work in cucumber, maize, and Arabidopsis shows variation in fluence-rate response and wavelength sensitivity (Casati and Walbot, 2004; Shinkle et al., 2004, 2009; Ulm et al., 2004). Molecular events are coordinated by changes in chromatin structure during acute and chronic exposure (Casati et al., 2008).

How are ultraviolet-B signals transduced? Ultraviolet-B can initiate molecular events by causing damage to DNA or from the generation of reactive oxygen species. Other events are mediated by specific activation of an Ultraviolet-B receptor and subsequent intracellular signal transmission. The UVR8 receptor was identified genetically. The UVR8 gene was first characterized in Arabidopsis, isolated as a mutant with lower flavonoid content and CHS transcript accumulation in response to ultraviolet-B irradiation that retained normal responses to other wavelengths (Kliebenstein et al., 2002). Constructs connecting UVR8 to the GFP reporter have shown that the fusion protein moves to the nucleus upon treatment with ultraviolet light, suggesting translocation as a step in signal transduction (Kaiserli and Jenkins, 2007). It was later shown that UVR8 dimerizes in vivo and dissociates into monomers that interact with COP1, the central repressor of light signaling (Rizzini et al., 2011). The physiological outcomes include changes in gene regulation, pigmentation, flavonoid biosynthesis, leaf expansion, and increased photosynthetic efficiency, among other processes (reviewed in Jenkins, 2014).

In terms of practical application in the control of plant growth in controlled environments, ultraviolet-B illumination and
UVR8-mediated signaling may provide special opportunities. While solid-state lighting in the ultraviolet spectrum remains expensive, it is becoming increasingly available and may be a useful addition to lighting systems. Ultraviolet-B is especially potent in increasing flavonoids and increasing the purple pigmentation of crops able to produce it. The ultraviolet-B effects on stem elongation and other processes suggest that ultraviolet-B could offer interesting opportunities to control growth without using photosynthetic radiation. The most interesting results may be from analysis of metabolites accumulating in response to ultraviolet-B treatment, as several pathways are known to be activated after ultraviolet-B treatment (Jansen et al., 1998).

\section{PREDICTED GREEN LIGHT RECEPTORS}

While the receptors themselves have not been identified, there is evidence that supports the hypothesis that green wavebands exert effects on discrete plant processes that are independent of known photosensors. Greenlight effects share several attributes in that they typically oppose the action of red or blue lightregulated plant processes (Wang and Folta, 2013). In the parlance of plant whispering, the use of green wavebands may be important in regulating plant stature, and certainly there is a role for affecting pigmentation.

The inhibitory effects of green wavebands were reported in the mid twentieth century by Frits Went (1957). Tomato seedlings grown under different fluence rates of red and blue light would reach a certain dry weight after a few weeks of growth. When plants were grown under visible light including a green component, the dry mass was lower, no matter how much the total fluence rate increased. The inhibition was not observed when a lavender filter was used, suggesting that the green component opposed the blue and red components (Went, 1957). Klein et al. (1965) would later identify various lines of evidence that suggested that green wavebands were not just less informative through blue and red light sensors, they were actively inhibitory (Klein, 1992; Klein et al., 1965).

While cryptochrome activation is reverted by green light, other responses appear to operate independently. For instance, researchers at NASA would examine the role of green light in plant growth. It was shown that green light changed stomatal conductance, leaf size and thickness (Kim et al., 2004a, 2004b). The decrease in stomatal conductance may be at least in part of the green light effect on stomatal opening, as green was shown to stop blue-light induced stomatal opening (Frechilla et al., 2000). The effect on leaf size could likely be ascribed to green-light-induced shade avoidance symptoms (Zhang et al., 2011). Green wavebands have also been shown to speed early stem growth (Folta, 2004) and decrease chloroplast transcript accumulation (Dhingra et al., 2006). Pigmentation in lettuces was 
decreased by the addition of green light to blue and red backgrounds (Zhang and Folta, 2012). In general, the effects of green wavebands are most conspicuous under lowfluence rate conditions, and they tend to oppose the effects of red and blue light. The green-sensing systems may also be useful in manipulating plant traits, as they may reduce the effects of cryptochromes while not affecting blue-light generated flux through phototropins. These wavelengths might be useful in balancing blue-light effects to change elongation growth and pigmentation in crop plants.

\section{CHLOROPHYLL AND CAROTENOIDS}

While not protein-based receptors, these molecules are activated by photons and exert effects on plant physiology. Energy intercepted through these pigments is typically funneled to photosystems to initiate photosynthesis. Excess energy results in a series of welldescribed fates that also must be considered in any light-integration model.

In terms of designing light recipes for matching photosynthesis to plant developmental cues imparted through the photosensors, chlorophyll and carotenoids offer an opportunity to charge the photosynthetic system without optimally affecting the receptors and attendant pathways that mediate development. In other words, the 500-600 wavebands still represent photosynthetically active radiation $(P A R)$, yet they are less efficient at driving processes that are phytochrome, cryptochrome, or phototropin mediated. This separation of $P A R$, from what might be termed "developmentally active radiation" could be a means to manipulate plant stature, color, nutrient content, and other plant attributes.

\section{COACTION}

It has long been noted that combinations of wavelengths behave additively, or in some cases synergistically. In other cases, the activation of more than one sensory system is required for the response (reviewed in Mohr, 1994). This phenomenon of "coaction" is defined as the functional interaction of activated light-sensory pathways.

In the application of narrow-bandwidth illumination to plant production, the role of coaction cannot be understated. Enhanced levels of trait control may be obtained by the activation of multiple photosensory systems. The accumulation of anthocyanin pigments is an excellent example that has been well described in many systems. Milo seedlings show an ultraviolet-inducible, far red reversible accumulation (Oelmüller and Mohr, 1985). On the other hand, kale sprouts show a higher anthocyanin accumulation when blue and far red light are applied together, more than any wavelength alone, or any combination with red light (Carvalho and Folta, 2014b). Several studies have described the potential manipulation via coaction through the use of lighting units featuring multiple emitters that may be independently controlled (Folta et al., 2005; Yano and Fujiwara, 2012).

\section{CONCLUSION}

The strategic manipulation of plant growth, development, physiology, metabolism, and behavior with light is predicated on an understanding of how light signals are sensed. The bottleneck in the process is the suite of photosensors that have discrete sensitivities to signals from the light environment. Connecting photons to traits via light signaling networks allows us to design custom light systems to stretch genetic potential and control plant product quantity, quality, and production timing. Selective activation of specific light-sensing pathways will allow growers to not simply grow plants, but to control how they grow.

\section{Literature Cited}

Ahmad, M. and A.R. Cashmore. 1993. HY4 gene of A. thaliana encodes a protein with characteristics of a blue-light photoreceptor. Nature 366:162-166.

Ahmad, M., N. Grancher, M. Heil, R.C. Black, B. Giovani, P. Galland, and D. Lardemer. 2002. Action spectrum for cryptochromedependent hypocotyl growth inhibition in Arabidopsis. Plant Physiol. 129:774-785.

Banerjee, R., E. Schleicher, S. Meier, R. Munoz Viana, R. Pokorny, M. Ahmad, R. Bittl, and A. Batschauer. 2007. The signaling state of Arabidopsis cryptochrome 2 contains flavin semiquinone. J. Biol. Chem. 282:14916-14922.

Boccalandro, H.E., C.V. Giordano, E.L. Ploschuk, P.N. Piccoli, R. Bottini, and J.J. Casal. 2012. Phototropins but not Cryptochromes mediate the blue light-specific promotion of stomatal conductance, while both enhance photosynthesis and transpiration under full sunlight. Plant Physiol. 158:1475-1484.

Bouly, J.P., E. Schleicher, M. Dionisio-Sese, F. Vandenbussche, D. Van der Straeten, N. Bakrim, S. Meier, A. Batschauer, P. Galland, R. Bittl, and M. Ahmad. 2007. Cryptochrome blue-light photoreceptors are activated through interconversion of flavin redox states. J. Biol. Chem. 282:93839391.

Briggs, W.R. and J.M. Christie. 2002. Phototropins 1 and 2: Versatile plant blue-light receptors. Trends Plant Sci. 7:204-210.

Brosche, M. and A. Strid. 2003. Molecular events following perception of ultraviolet-B radiation by plants. Physiol. Plant. 117:1-10.

Canamero, R., N. Bakrim, J.-P. Bouly, A. Garay, E. Dudkin, Y. Habricot, and M. Ahmad. 2006. Cryptochrome photoreceptors cry1 and cry2 antagonistically regulate primary root elongation in Arabidopsis thaliana. Planta 224:9951003.

Carvalho, S. and K. Folta. 2014a. Environmentally modified organisms-extracting genetic potential withlight. Crit. Rev. Plant Sci. (Submitted).

Carvalho, S.D. and K.M. Folta. 2014b. Sequential light programs shape kale (Brassica napus) sprout appearance and alter metabolic and nutrient content. Horticulture Research 1:1-13.

Casati, P., M. Campi, F. Chu, N. Suzuki, D. Maltby, S. Guan, A.L. Burlingame, and V. Walbot. 2008. Histone acetylation and chromatin remodeling are required for UV-B-dependent transcriptional activation of regulated genes in maize. Plant Cell 20:827-842.
Casati, P. and V. Walbot. 2004. Rapid transcriptome responses of maize (Zea mays) to UV-B in irradiated and shielded tissues. Genome Biol. 5.

Chatterjee, M., P. Sharma, and J.P. Khurana. 2006. Cryptochrome 1 from Brassica napus is upregulated by blue light and controls hypocotyl/ stem growth and anthocyanin accumulation. Plant Physiol. 141:61-74.

Chen, F., B. Li, G. Li, J.-B. Charron, M. Dai, X. Shi, and X.W. Deng. 2014. Arabidopsis Phytochrome A directly targets numerous promoters for individualized modulation of genes in a wide range of pathways. The Plant Cell Online.

Chen, F., X. Shi, L. Chen, M. Dai, Z. Zhou, Y. Shen, J. Li, G. Li, N. Wei, and X.W. Deng. 2012. Phosphorylation of FAR-RED ELONGATED HYPOCOTYL1 Is a Key Mechanism Defining Signaling Dynamics of Phytochrome A under Red and Far-Red Light in Arabidopsis. The Plant Cell Online 24:1907-1920.

Chen, H., X. Huang, G. Gusmaroli, W. Terzaghi, O.S. Lau, Y. Yanagawa, Y. Zhang, J. Li, J.-H. Lee, and D. Zhu. 2010. Arabidopsis CULLIN4damaged DNA binding protein 1 interacts with CONSTITUTIVELY PHOTOMORPHOGENIC1SUPPRESSOR OF PHYA complexes to regulate photomorphogenesis and flowering time. The Plant Cell Online 22:108-123.

Chen, M., J. Chory, and C. Fankhauser. 2004. Light signal transduction in higher plants. Annu. Rev. Genet. 38:87-117.

Chia, P.-L. and C. Kubota. 2010. End-of-day farred light quality and dose requirements for tomato rootstock hypocotyl elongation. HortScience 45:1501-1506.

Christie, J.M., P. Reymond, G.K. Powell, P. Bernasconi, A.A. Raibekas, E. Liscum, and W.R. Briggs. 1998. Arabidopsis NPH1: A flavoprotein with the properties of a photoreceptor for phototropism. Science 282:1698-1701.

Devlin, P.F. and S.A. Kay. 2000. Cryptochromes are required for phytochrome signaling to the circadian clock but not for rhythmicity. Plant Cell 12:2499-2510.

Dhingra, A., D.H. Bies, K.R. Lehner, and K.M. Folta. 2006. Green light adjusts the plastid transcriptome during early photomorphogenic development. Plant Physiol. 142:1256-1266.

Duek, P.D., M.V. Elmer, V.R. van Oosten, and C. Fankhauser. 2004. The degradation of HFR1, a putative bHLH class transcription factor involved in light signaling, is regulated by phosphorylation and requires COP1. Curr. Biol. 14:2296-2301.

El-Assal, S.E.D., C. Alonso-Blanco, C.J. Hanhart, and M. Koornneef. 2004. Pleiotropic effects of the Arabidopsis cryptochrome 2 allelic variation underlie fruit trait-related QTL. Plant Biol. 6:370-374.

Feng, S., C. Martinez, G. Gusmaroli, Y. Wang, J. Zhou, F. Wang, L. Chen, L. Yu, J.M. Iglesias-Pedraz, and S. Kircher. 2008. Coordinated regulation of Arabidopsis thaliana development by light and gibberellins. $\mathrm{Na}-$ ture 451:475-479.

Folta, K.M. 2004. Green light stimulates early stem elongation, antagonizing light-mediated growth inhibition. Plant Physiol. 135:1407-1416.

Folta, K.M. and K.S. Childers. 2008. Light as a growth regulator: Controlling plant Biology with narrow-bandwidth solid-state lighting systems. HortScience 43:1957-1964.

Folta, K.M. and L.S. Kaufman. 2003. Phototropin 1 is required for high-fluence blue-light-mediated mRNA destabilization. Plant Mol. Biol. 51:609618.

Folta, K.M., L. Koss, R. McMorrow, H.H. Kim, J.D. Kenitz, R. Wheeler, and J.C. Sager. 2005. 
Design and fabrication of LED-based light arrays for plant research. BMC Plant Biol. 5:17-28.

Folta, K.M. and E.P. Spalding. 2001. Unexpected roles for cryptochrome 2 and phototropin revealed by high-resolution analysis of blue light-mediated hypocotyl growth inhibition. Plant J. 26:471-478.

Fox, A., G. Soto, A. Jones, J. Casal, J. Muschietti, and M. Mazzella. 2012. cry1 and GPA1 signaling genetically interact in hook opening and anthocyanin synthesis in Arabidopsis. Plant Mol. Biol. 80:315-324.

Frechilla, S., L.D. Talbott, R.A. Bogomolni, and E. Zeiger. 2000. Reversal of blue light-stimulated stomatal opening by green light. Plant Cell Physiol. 41:171-176.

Frohnmeyer, H. and D. Staiger. 2003. Ultraviolet-B radiation-mediated responses in plants. Balancing damage and protection. Plant Physiol. 133:1420-1428.

Furuya, M. and J.G. Torrey. 1964. The reversible inhibition by red and far-red light of auxininduced lateral root initiation in isolated pea roots. Plant Physiol. 39:987-991.

Galland, P. and N. Tolle. 2003. Light-induced fluorescence changes in Phycomyces: Evidence for blue light-receptor associated flavosemiquinones. Planta 217:971-982.

Giliberto, L., G. Perrotta, P. Pallara, J.L. Weller, P.D. Fraser, P.M. Bramley, A. Fiore, M. Tavazza, and G. Giuliano. 2005. Manipulation of the blue light photoreceptor cryptochrome 2 in tomato affects vegetative development, flowering time, and fruit antioxidant content. Plant Physiol. 137:199-208.

Gracheva, E.O., J.F. Cordero-Morales, J.A. GonzalezCarcacia, N.T. Ingolia, C. Manno, C.I. Aranguren, J.S. Weissman, and D. Julius. 2011. Ganglionspecific splicing of TRPV1 underlies infrared sensation in vampire bats. Nature 476:88-91.

Gracheva, E.O., N.T. Ingolia, Y.M. Kelly, J.F. Cordero-Morales, G. Hollopeter, A.T. Chesler, E.E. Sanchez, J.C. Perez, J.S. Weissman, and D. Julius. 2010. Molecular basis of infrared detection by snakes. Nature 464:1006-1011.

Guo, H., H. Yang, T.C. Mockler, and C. Lin. 1998. Regulation of flowering time by Arabidopsis photoreceptors. Science 279:1360-1363.

Hamner, M., E. Kowalik, K. Folta, and J. Shinkle. 2009. Evidence of physiological action of phototropin1 in response to UV-C illumination. Comparative Biochemistry and Physiology A-Molecular \& Integrative Physiology 153A: S200-S201.

Hirose, F., T. Shinomura, T. Tanabata, H. Shimada, and M. Takano. 2006. Involvement of rice cryptochromes in de-etiolation responses and flowering. Plant Cell Physiol. 47:915-925.

Ilias, I.F. and N. Rajapakse. 2005. The effects of end-of-the-day red and far-red light on growth and flowering of Petunia xhybrida 'Countdown Burgundy' grown under photoselective films. HortScience 40:131-133.

Inoue, S.-i., T. Kinoshita, A. Takemiya, M. Doi, and K.-i. Shimazaki. 2008. Leaf positioning of Arabidopsis in response to blue light. Mol. Plant 1:15-26.

Islam, M.A., G. Kuwar, J.L. Clarke, D.-R. Blystad, H.R. Gislerød, J.E. Olsen, and S. Torre. 2012. Artificial light from light emitting diodes (LEDs) with a high portion of blue light results in shorter poinsettias compared to high pressure sodium (HPS) lamps. Sci. Hort. 147:136-143.

Jansen, M.A.K., V. Gaba, and B.M. Greenberg. 1998. Higher plants and UV-B radiation: Balancing damage, repair and acclimation. Trends Plant Sci. 3:131-135.
Jenkins, G.I. 2014. The UV-B Photoreceptor UVR8: From structure to physiology. The Plant Cell Online 26:21-37.

Johnson, C.F., C.S. Brown, R.M. Wheeler, J.C. Sager, D.K. Chapman, and G.F. Deitzer. 1996. Infrared light-emitting diode radiation causes gravitropic and morphological effects in darkgrown oat seedlings. Photochem. Photobiol. 63:238-242.

Kadomura-Ishikawa, Y., K. Miyawaki, S. Noji, and A. Takahashi. 2013. Phototropin 2 is involved in blue light-induced anthocyanin accumulation in Fragaria $\mathrm{x}$ ananassa fruits. J. Plant Res. 126:847-857.

Kagawa, T. 2003. The phototropin family as photoreceptors for blue light-induced chloroplast relocation. J. Plant Res. 116:77-82.

Kaiserli, E. and G.I. Jenkins. 2007. UV-B Promotes rapid nuclear translocation of the Arabidopsis UV-B specific signaling component UVR8 and activates its function in the nucleus. The Plant Cell Online 19:2662-2673.

Kasperbauer, M.J. and D.E. Peaslee. 1973. Morphology and photosynthetic efficiency of tobacco leaves that received end-of-day red and far red light during development. Plant Physiol. $52: 440-442$

Kaufman, L.S., W.R. Briggs, and W.F. Thompson. 1985. Phytochrome control of specific mRNA levels in developing pea buds: The presence of both very low fluence and low fluence responses. Plant Physiol. 78:388-393.

Keuskamp, D.H., R. Sasidharan, I. Vos, A.J.M Peeters, L.A.C.J. Voesenek, and R. Pierik. 2011. Blue-light-mediated shade avoidance requires combined auxin and brassinosteroid action in Arabidopsis seedlings. Plant $\mathrm{J}$. 67:208-217.

Khanna, R., Y. Shen, C.M. Marion, A. Tsuchisaka, A. Theologis, E. Schäfer, and P.H. Quail. 2007. The basic helix-loop-helix transcription factor PIF5 acts on ethylene biosynthesis and phytochrome signaling by distinct mechanisms. The Plant Cell Online 19:3915-3929.

Khattak, A. and S. Pearson. 2006. Spectral filters and temperature effects on the growth and development of chrysanthemums under low light integral. Plant Growth Regulat. 49:61-68.

Kilian, J., D. Whitehead, J. Horak, D. Wanke, S. Weinl, O. Batistic, C. D'Angelo, E. Bornberg-Bauer, J. Kudla, and K. Harter. 2007. The AtGenExpress global stress expression data set: Protocols, evaluation and model data analysis of UV-B light, drought and cold stress responses. Plant J. 50:347-363

Kim, H.H., G.D. Goins, R.M. Wheeler, and J.C. Sager. 2004a. Green light supplementation for enhanced lettuce growth under red and blue light-emitting diodes. HortScience 39:16171622.

Kim, H.H., G.D. Goins, R.M. Wheeler, and J.C. Sager. 2004b. Stomatal conductance of lettuce grown under or exposed to different light quality. Ann. Bot. (Lond.) 94:91-97.

Klein, R.M. 1992. Effects of green light on biological systems. Biol. Rev. Camb. Philos. Soc. 67:199-284.

Klein, R.M., P.C. Edsall, and A.C. Gentile. 1965 Effects of near ultraviolet and green radiations on plant growth. Plant Physiol. 40:903-906.

Kleine, T., P. Kindgren, C. Benedict, L. Hendrickson, and A. Strand. 2007. Genome-wide gene expression analysis reveals a critical role for CRYPTOCHROME1 in the response of Arabidopsis to high irradiance. Plant Physiol. 144:1391-1406.

Kliebenstein, D.J., J.E. Lim, L.G. Landry, and R.L. Last. 2002. Arabidopsis UVR8 regulates
ultraviolet-B signal transduction and tolerance and contains sequence similarity to human regulator of chromatin condensation 1. Plant Physiol. 130:234-243.

Kozuka, T., N. Suetsugu, M. Wada, and A. Nagatani. 2013. Antagonistic regulation of leaf flattening by Phytochrome B and Phototropin in Arabidopsis thaliana. Plant Cell Physiol. 54:69-79.

Leivar, P., E. Monte, Y. Oka, T. Liu, C. Carle, A. Castillon, E. Huq, and P.H. Quail. 2008. Multiple Phytochrome-interacting bHLH transcription factors repress premature seedling photomorphogenesis in darkness. Curr. Biol. 18:1815-1823.

Lin, C. 2000. Plant blue-light receptors. Trends Plant Sci. 5:337-342.

Lin, C. 2002. Blue light receptors and signal transduction. Plant Cell 14:S207-S225.

Liu, H., Q. Wang, Y. Liu, X. Zhao, T. Imaizumi, D.E. Somers, E.M. Tobin, and C. Lin. 2013. Arabidopsis CRY2 and ZTL mediate blue-light regulation of the transcription factor CIB1 by distinct mechanisms. Proc. Natl. Acad. Sci. USA 110:17582-17587.

Loughrin, J.H. and M.J. Kasperbauer. 2003. Aroma content of fresh basil (Ocimum basilicum L.) leaves is affected by light reflected from colored mulches. J. Agr. Food Chem. 51:22722276.

Lund, J.B., T.J. Blom, and J.M. Aaslyng. 2007. End-of-day lighting with different red/far-red ratios using light-emitting diodes affects plant growth of Chrysanthemum $\times$ morifolium Ramat. 'Coral Charm'. HortScience 42:1609_ 1611

Mao, J., Y.C. Zhang, Y. Sang, Q.H. Li, and H.Q Yang. 2005. From the cover: A role for Arabidopsis cryptochromes and COP1 in the regulation of stomatal opening. Proc. Natl. Acad. Sci. USA 102:12270-12275.

Martinez-Garcia, J.F., E. Huq, and P.H. Quail 2000. Direct targeting of light signals to a promoter element-bound transcription factor. Science 288:859-863.

Mohr, H. 1962. Primary effects of light on growth. Annu. Rev. Plant Physiol. 13:465-488.

Mohr, H. 1994. Coaction between pigment systems, p. 353-373. In: R.E. Kendrick and G.H.M. Kronenberg (eds.). Photomorphogenesis in Plants. Springer, Netherlands.

Moni, A., A.Y. Lee, W.R. Briggs, and I.S. Han. 2014. The blue light receptor Phototropin 1 suppresses lateral root growth by controlling cell elongation. Plant Biology 17:34-40.

Moreno, J.E., Y. Tao, J. Chory, and C.L. Ballaré. 2009. Ecological modulation of plant defense via phytochrome control of jasmonate sensitivity. Proc. Natl. Acad. Sci. USA 106:4935-4940.

Nelson, D.C., J. Lasswell, L.E. Rogg, M.A. Cohen, and B. Bartel. 2000. FKF1, a clock-controlled gene that regulates the transition to flowering in Arabidopsis. Cell 101:331-340.

Oelmüller, R. and H. Mohr. 1985. Mode of coaction between blue/UV light and light absorbed by phytochrome in light-mediated anthocyanin formation in the milo (Sorghum vulgare Pers.) seedling. Proc. Natl. Acad. Sci. USA 82:6124-6128.

Osterlund, M.T., C.S. Hardtke, N. Wei, and X.W. Deng. 2000. Targeted destabilization of HY5 during light-regulated development of Arabidopsis. Nature 405:462-466.

Piskurewicz, U., V. Turečková, E. Lacombe, and L. Lopez-Molina. 2009. Far-red light inhibits germination through DELLA-dependent stimulation of ABA synthesis and ABI3 activity. EMBO J. 28:2259-2271. 
Pjon, C.-J. and M. Furuya. 1967. Phytochrome action in Oryza sativa LI Growth responses of etiolated coleoptiles to red, far-red and blue light. Plant Cell Physiol. 8:709-718.

Platten, J.D., E. Foo, F. Foucher, V. Hecht, J.B. Reid, and J.L. Weller. 2005. The cryptochrome gene family in pea includes two differentially expressed CRY2 genes. Plant Mol. Biol. 59:683-696.

Reed, J.W., P. Nagpal, D.S. Poole, M. Furuya, and J. Chory. 1993. Mutations in the gene for the red/far-red light receptor phytochrome B alter cell elongation and physiological responses throughout Arabidopsis development. Plant Cell 5:147-157.

Rizzini, L., J.-J. Favory, C. Cloix, D. Faggionato, A. O'Hara, E. Kaiserli, R. Baumeister, E. Schäfer, F. Nagy, G.I. Jenkins, and R. Ulm. 2011. Perception of UV-B by the Arabidopsis UVR8 protein. Science 332:103-106.

Sancar, A. 2003. Structure and function of DNA photolyase and cryptochrome blue-light photoreceptors. Chem. Rev. 103:2203-2238.

Schultz, T.F., T. Kiyosue, M. Yanovsky, M. Wada, and S.A. Kay. 2001. A role for LKP2 in the circadian clock of Arabidopsis. Plant Cell 13:2659-2670.

Sharrock, R.A. and T. Clack. 2004. Heterodimerization of type II phytochromes in Arabidopsis. Proc. Natl. Acad. Sci. USA 101:11500-11505.

Shinkle, J.R., A.K. Atkins, E.E. Humphrey, C.W. Rodgers, S.L. Wheeler, and P.W. Barnes. 2004. Growth and morphological responses to different UV wavebands in cucumber (Cucumis sativum) and other dicotyledonous seedlings. Physiol. Plant. 120:240-248.

Shinkle, J.R., M.C. Edwards, A. Koenigh, A. Shaltz, and P.W. Barnes. 2009. Photomorphogenic regulation of increases in UV absorbing pigments in cucumber (Cucumis sativus) and Arabidopsis thaliana seedlings induced by different UV-B and UV-C wavebands. Physiol. Plant. (in press).
Somers, D.E., P.F. Devlin, and S.A. Kay. 1998. Phytochromes and cryptochromes in the entrainment of the Arabidopsis circadian clock. Science 282:1488-1490.

Somers, D.E., T.F. Schultz, M. Milnamow, and S.A. Kay. 2000. ZEITLUPE encodes a novel clock-associated PAS protein from Arabidopsis. Cell 101:319-329.

Song, Y.H., D.A. Estrada, R.S. Johnson, S.K. Kim, S.Y. Lee, M.J. MacCoss, and T. Imaizumi. 2014. Distinct roles of FKF1, GIGANTEA, and ZEITLUPE proteins in the regulation of CONSTANS stability in Arabidopsis photoperiodic flowering. Proc. Natl. Acad. Sci. USA 111:17672-17677.

Song, Y.H., R.W. Smith, B.J. To, A.J. Millar, and T. Imaizumi. 2012. FKF1 conveys timing information for CONSTANS stabilization in photoperiodic flowering. Science 336:10451049.

Spalding, E.P. and K.M. Folta. 2005. Illuminating topics in plant photobiology. Plant Cell Environ. 28:39-53.

Stamps, R.H. 2009. Use of colored shade netting in horticulture. HortScience 44:239-241.

Takeda, F., D.M. Glenn, A. Callahan, J. Slovin, and G.W. Stutte. 2010. Delaying flowering in shortday strawberry transplants with photoselective nets. Intl. J. Fruit Sci. 10:134-142.

Takemiya, A., S.-i. Inoue, M. Doi, T. Kinoshita, and K.-i. Shimazaki. 2005. Phototropins promote plant growth in response to blue light in low light environments. The Plant Cell Online 17:1120-1127.

Tao, Y., J.-L. Ferrer, K. Ljung, F. Pojer, F. Hong, J.A. Long, L. Li, J.E. Moreno, M.E. Bowman, and L.J. Ivans. 2008. Rapid synthesis of auxin via a new tryptophan-dependent pathway is required for shade avoidance in plants. Cell 133:164-176.

Ulm, R., A. Baumann, A. Oravecz, Z. Mate, E. Adam, E.J. Oakeley, E. Schafer, and F. Nagy. 2004. Genome-wide analysis of gene expression reveals function of the bZIP transcription factor HY5 in the UV-B response of
Arabidopsis. Proc. Natl. Acad. Sci. USA 101:13971402.

Valverde, F., A. Mouradov, W. Soppe, D. Ravenscroft, A. Samach, and G. Coupland. 2004. Photoreceptor regulation of CONSTANS protein in photoperiodic flowering. Science 303:1003-1006.

Vince, D. 1964. Photomorphogenesis in plant stems. Biol. Rev. Camb. Philos. Soc. 39:506533.

Vince, D., R. Spencer, and J. Blake. 1964. Some effects of wave-length of supplementary light on photoperiodic behaviour of long-day plants carnation + lettuce. Physiol. Plant. 17:119.

Wang, Y. and K.M. Folta. 2013. Contributions of green light to plant growth and development. Amer. J. Bot. 100:70-78.

Went, F.W. 1957. The Experimental Control of Plant Growth. Chronica Botanica, Waltham, MA.

Yamazaki, K., Y. Ishii, and I. Tanaka. 2007. Spectral sensitivity of the promotion and inhibition of flowering in morning glory (Pharbitis nil). Envrion. Control Biol. 45:75-83.

Yang, Z.-C., C. Kubota, P.-L. Chia, and M. Kacira 2012. Effect of end-of-day far-red light from a movable LED fixture on squash rootstock hypocotyl elongation. Sci. Hort. 136:81-86.

Yano, A. and K. Fujiwara. 2012. Plant lighting system with five wavelength-band lightemitting diodes providing photon flux density and mixing ratio control. Plant Methods 8:46.

Zhang, T. and K. Folta. 2012. Green light signaling and response. Plant Signal. Behav. 7:1-4.

Zhang, T., S.A. Maruhnich, and K.M. Folta. 2011. Green light induces shade avoidance symptoms. Plant Physiol. 157:1528-1536.

Zhu, D., A. Maier, J.-H. Lee, S. Laubinger, Y. Saijo, H. Wang, L.-J. Qu, U. Hoecker, and X.W. Deng. 2008. Biochemical characterization of Arabidopsis complexes containing CONSTITUTIVELY PHOTOMORPHOGENIC1 and SUPPRESSOR OF PHYA proteins in light control of plant development. The Plant Cell Online 20:23072323. 